\title{
Effect of Prefilmer Edge Thickness on Breakup Phenomena of Liquid Film in Prefilming Airblast Atomizer
}

\author{
Takahiro Okabe $^{\star 1}$, Naoki Katagata ${ }^{1}$, Toshihiro Sakaki ${ }^{1}$, Takao Inamura ${ }^{1}$, Koji Fumoto ${ }^{1}$ \\ ${ }^{1}$ Graduate School of Science and Technology, Hirosaki University, Japan \\ ${ }^{\star}$ Corresponding author: oka@hirosaki-u.ac.jp
}

\begin{abstract}
This paper describes the investigation of the effect of a prefilmer edge thickness on the breakup phenomena of a liquid film in a prefilmer airblast atomizer. The breakup phenomena of the liquid film at five prefilmer edge thicknesses $(160,500,1250,2000$, and $3000 \mu \mathrm{m})$ under various conditions was observed using a high-speed camera. The breakup length of the liquid film was calculated by an image processing technique developed in this study. In order to quantitatively evaluate the effect of the prefilmer edge thickness on the breakup frequency, the Fast Fourier Transformation (FFT) analysis was conducted based on the time evolution of the breakup length. The results indicated that the breakup length increase and the breakup frequency decreases by increasing prefilmer edge thickness due to a larger volume of a liquid accumulation attaching to the prefilmer edge. The FFT analysis showed that the increase in prefilmer edge thickness causes the transition of the maximal power spectrum to a lower frequency (i.e. less than $100 \mathrm{~Hz}$ ) due to the increase in the liquid accumulation at the edge as well. Finally, a dimensionless correlation has been proposed for the breakup length of a liquid film.
\end{abstract}

\section{Keywords}

Prefilming airblast atomizer, Breakup length, Breakup frequency, Prefilmer edge thickness, FFT analysis

\section{Introduction}

In the recent jet engine, the prefilming airblast atomizer has been widely used for the liquid fuel injection due to a good spray characteristics even at low fuel injection pressure. This type of atomizer uses the high-speed air stream flowing in the combustor for the atomization of a liquid fuel. Thus, the pressure loss of atomizing air in an atomizer is smaller than a conventional atomizer using the air stream, and leads to stable performances of the combustor over the entire range of operating conditions.

The atomization mechanism of a prefilming type of airblast atomizer is well known. After injection, the liquid homogeneously wet the prefilmer surface, and flows down to the prefilmer edge in the form of a liquid film that is sheared by the high-speed air stream. The liquid film accumulates at the prefilmer edge. This accumulation is sheared by air and disintegrated into the bag-shaped liquid framed by a thicker rim. The bag-shaped part of the structure bursts and generates fine droplets. The rim is stretched and fragments into two elongated ligaments that disintegrate into larger droplets.

Many researchers have studied the spray characteristics of a prefilming type of airblast atomizer, and revealed that a liquid film thickness, liquid flowrate, and air velocity are important parameters. Lefebvre reviewed the investigations on airblast atomizers, and pointed out the advantages of airblast atomizers such as lower fuel pressure, low soot formation, constant fuel distribution over the whole range of fuel flow rate, and fine mixing between fuel and air [1]. Inamura et al. experimentally investigated the spray characteristics of a prefilming type of airblast atomizer using the two-dimensional test atomizer with a rectangular cross-section [2]. Furthermore, they proposed new breakup model of a liquid film at a prefilmer edge based on the Kelvin-Helmholtz and Rayleigh-Taylor instability. The predicted wavelength, ligament diameter and mean droplet diameter were compared with the measurements. The predicted SMDs showed good agreements with the measurements at small liquid flowrate, but it showed slightly smaller values than the measurements at large liquid flowrate.

However, little research on the effect of the geometric shape of a prefilmer on the spray characteristics of prefilming airblast atomizer is currently available. Gepperth et al. conducted the experiments to investigate the effect of liquid physical properties, film flow rate, and prefilming configurations on primary atomization of liquid film. Their experimental results showed that mean air velocity and prefilmer edge thickness were found to be the important parameters to the spray characteristics and the breakup phenomena of liquid film [3]. The prefilmer length, liquid physical properties and liquid volume flow rate seem to have only a minor effect on the mean droplet diameter but affect the ligament formation process. They pointed out three important parameters: the prefilmer edge thickness, the surface tension of the liquid, and the momentum flux of the gas. However, they did not investigate the effect of widely varying the prefilmer edge thickness on the breakup phenomena and spray characteristics. 
Furthermore, up to now there is no correlation which contains the effect of the prefilmer edge thickness on the characteristics of the breakup phenomena such as the breakup length and the breakup frequency, etc.

In this study, we focus on the prefilmer edge thickness as an important parameter, and aim to experimentally clarify the effect on the breakup phenomena of a liquid sheet in a prefilming airblast atomizer. In addition, we propose a dimensionless correlation for the breakup length of a liquid film which contains the effect of the prefilmer edge thickness.

\section{Experimental Apparatus}

Figure 1 (a) shows the experimental apparatus. The air generated by the blower is supplied to the contraction nozzle via a honeycomb. The test atomizer is attached to the exit of the contraction nozzle. The water in the pressure tank is pressurized by a high-pressure air from the compressor, and then it is supplied to the prefilmer in the test atomizer through a flow meter. The details of the experimental apparatus are presented in the previous paper [4]. Figure 1 (b) shows the test atomizer used in this study. The test atomizer is two-dimensional for easy observation of the breakup phenomena of a liquid film. The prefilmer is set at the center in the atomizer, and the porous metal was used at a liquid outlet part to uniform the liquid flowrate distribution in the traverse direction. In order to avoid an undesirable change in the air velocity in the test channel, the channel height is fixed to $10 \mathrm{~mm}$ for all prefilmers with different edge thicknesses. The breakup phenomena of a liquid film are observed by the high-speed video camera which is placed above the prefilmer edge. The metal halide lamp is used as the light source. The field of view is appropriately adjusted in accordance with the experimental conditions. The frame rate is also adjusted from 3000 to $10000 \mathrm{fps}$. In this study, tap water was used as the atomized liquid and air as the atomizing gas. The liquid volume flowrate per unit width of a prefilmer was ranged from 0.8 to $2.6 \mathrm{~cm} 3 /(\mathrm{s} \cdot \mathrm{cm})$, and the air velocity at the atomizer exit was ranged from 16.6 to $83.0 \mathrm{~m} / \mathrm{s}$. During the experiments, the film dry-out was not observed upstream the prefilmer edge except the case under $u_{g}=16.6 \mathrm{~m} / \mathrm{s}$. All experiments were conducted under the atmospheric pressure.

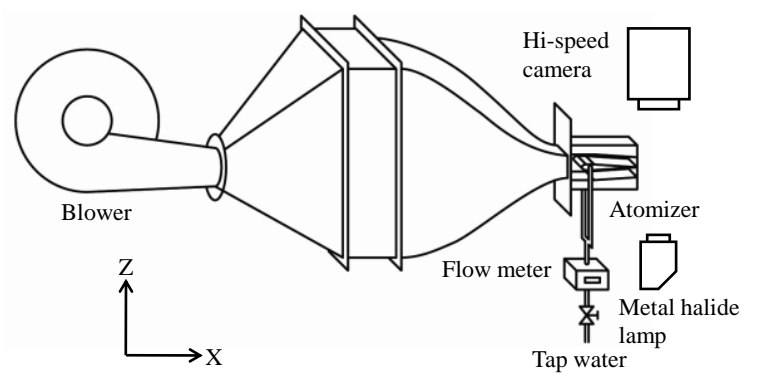

(a) Experimental apparatus
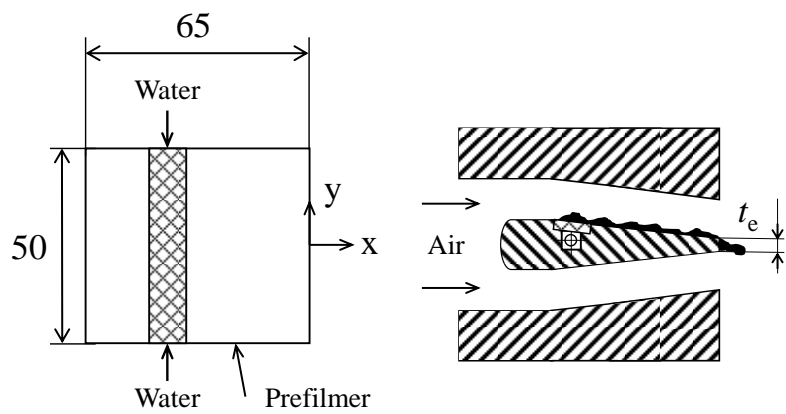

(b) Test atomizer.

Figure 1. Experimental apparatus.

\section{Breakup phenomena of liquid film}

Details of the effects of an air velocity and prefilmer edge thickness on the breakup phenomena of a liquid film at a prefilmer edge under liquid flowrate of $2.6 \mathrm{~cm}^{3} /(\mathrm{s} \cdot \mathrm{cm})$ are illustrated in Figure 2. In this study, five different prefilmer configurations have been tested to investigate the effect of widely varying prefilmer edge thickness $\left(t_{e}=160,500\right.$, $1250,2000$, and $3000 \mu \mathrm{m})$. The air and liquid flow from the bottom to the top in the photographs.

During the experiments, it can be observed that the breakup process basically consists of the three stages. First, a liquid film that was flowing over the prefilmer surface accumulates at the edge and in the wake region of the prefilmer edge. Second, the liquid accumulated at a prefilmer edge is then sheared by air and fragmented into bag-shaped liquid film and ligaments. As soon as this bag-shaped film bursts, fine droplets are generated. The ligaments remain attaching to the prefilmer edge, and disintegrates later into larger droplets.

The visual inspection showed a significant effect of the prefilmer edge thickness on the breakup phenomena of the liquid film such as the breakup length, the size and number of ligaments, and the liquid accumulation attaching to the prefilmer edge. As the prefilmer edge thickness increases, the breakup length of the liquid film and the size of ligaments increases. Due to these geometrical changes, larger droplets are generated. At investigating the liquid accumulation, it was observed that the accumulated volume of liquid clearly depends on the prefilmer edge thickness. It should be noted that, for the case of $t_{e}=160 \mu \mathrm{m}$ under larger air velocity $\left(u_{g}=66.7\right.$ and $\left.83.0 \mathrm{~m} / \mathrm{s}\right)$, no liquid accumulation was observed. Also, at the lower flowrate condition $\left(Q_{l}=0.8 \mathrm{~cm} 3 /(\mathrm{s} \cdot \mathrm{cm})\right)$, there was no liquid accumulation at the prefilmer edge under even a smaller air velocity $\left(u_{g}=32.7\right.$ and $\left.50.0 \mathrm{~m} / \mathrm{s}\right)$. On the other hand, it 
is obvious that increasing air velocity reduces the breakup length of the liquid film. In addition, it can be seen that the number of atomization events per second increases with increasing air velocities.

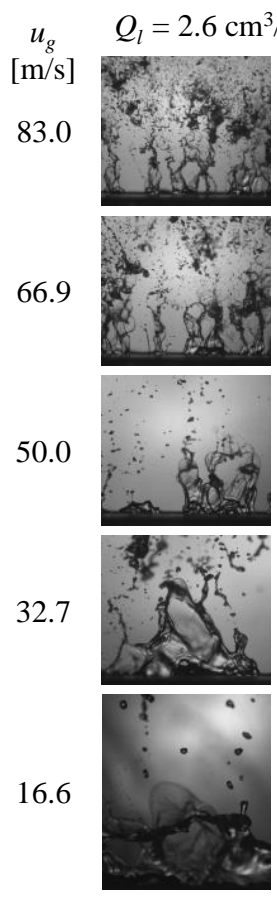

160
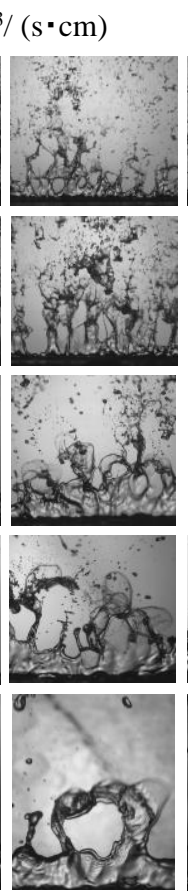

500
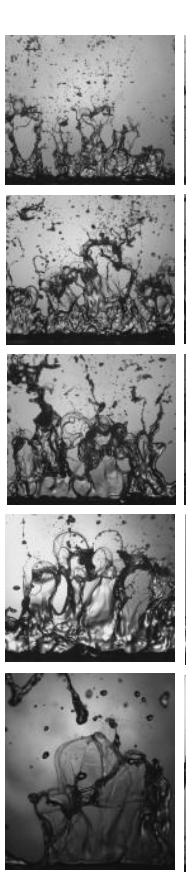

1250
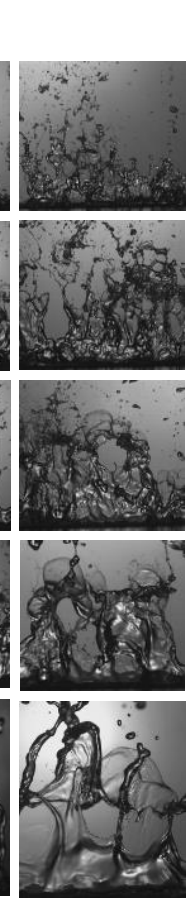

2000

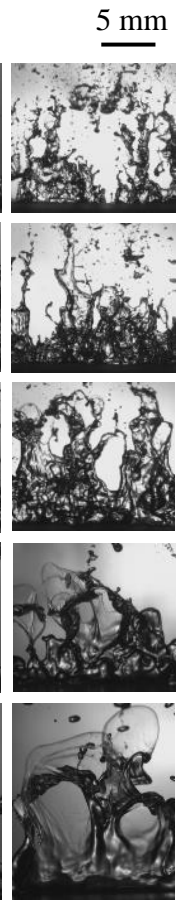

3000 $t_{e}$

Figure 2. Breakup phenomena of liquid film at each prefilmer edge thickness when $Q_{l}=2.6 \mathrm{~cm}^{3} /(\mathrm{s} \cdot \mathrm{cm})$.

\section{Image processing method}

To quantitatively evaluate the effect of the prefilmer edge thickness on the breakup phenomena of a liquid film, the image processing technique to determine the breakup length has been developed using MATLAB (Math Works) in this study.

Figure 3 shows the procedure of an image processing method developed in this study. From the original image at the prefilmer edge (a), a binary image (b) is generated. This binary image processing replaces each pixel in an image with a black pixel if the image intensity is less than the threshold, or a white pixel if it is greater than the threshold. Then, all the pixels framed by a white pixel are filled with white as shown in (c). The parts of droplets and liquid lumps are removed, and only the part of accumulated liquid film remains (d). The area $A_{b}$ is calculated by the number of pixels in the accumulated liquid film. Finally, based on the prefilmer edge width and the calculated area of the liquid film, the breakup length $L_{b}$ can be derived as follows:

$$
L_{b}=A_{b} / w,
$$

where $L_{b}$ is the mean breakup length, $A_{b}$ is the area of the liquid film, and $w$ is the width of the prefilmer edge. The red line in Figure 3 (d) indicates the mean breakup length which is the spatially-averaged value of the entire calculation domain. For each experimental condition, a set of 1000 images was processed using above mentioned method.
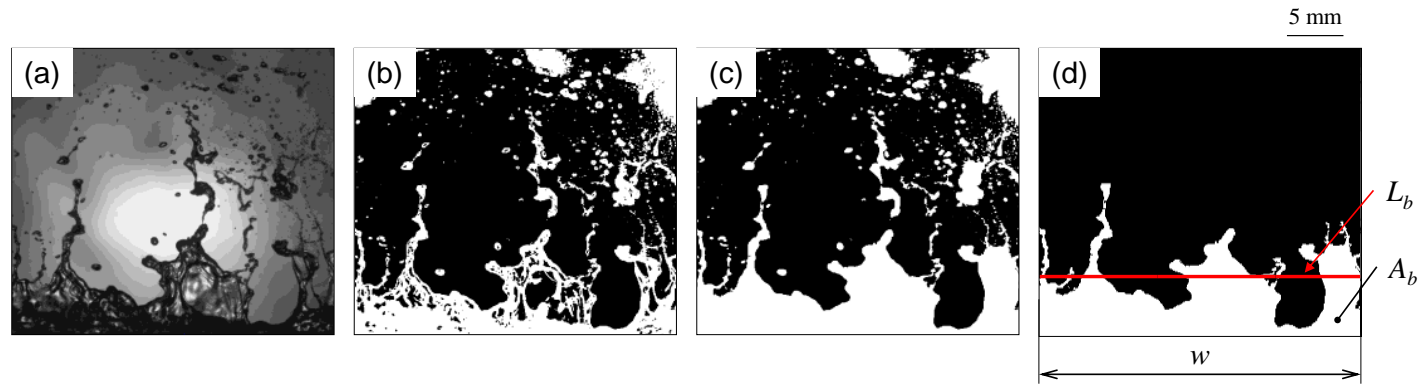

Figure 3. Procedure of image processing for determining breakup length of liquid film $L_{b}$. 


\section{Results and discussion}

Figure 4 shows the time evolution of the breakup length of a liquid film at each prefilmer edge thickness $\left(t_{e}=160\right.$, $500,1250,2000$, and $3000 \mu \mathrm{m}$ ) under (a) $u_{g}=32.7 \mathrm{~m} / \mathrm{s}$ and (b) $u_{g}=66.7 \mathrm{~m} / \mathrm{s}$, at $Q_{l}=2.6 \mathrm{~cm}^{3} /(\mathrm{s} \cdot \mathrm{cm})$. The red line in each graph indicates the time-averaged breakup length for 1000 images. The time variations of the breakup length show a sawtooth-shaped curve. The primary breakup is characterized by a sudden drop of this curve. This is similar to the simulation results by Koch et al. [5].

As the prefilmer edge thickness increases, the mean breakup length increases and the breakup frequency decreases. In conjunction with the visual observations shown in Figure 2, this seems to be due to the accumulated liquid at the prefilmer edge. Obviously, the liquid film was accumulated the atomizer edge before the primary disintegration. The accumulated liquid volume depends on the prefilmer edge thickness. A larger liquid volume is accumulated at a thicker atomizing edge, and results in the elongation of the liquid film attaching to the edge. This is consistent with the previous studies (Gepperth et al. [3], and Koch et al. [5]). In addition, Sattelmayer and Wittig concluded that the characteristics of the wavy liquid film on the prefilmer such as the film velocity, film thickness, and breakup frequency have a weak impact on the breakup process due to a suppression by the liquid accumulation at the prefilmer edge [6]. Also, it is obvious that the maximum breakup length, which means the elongation of the liquid film, became larger as the prefilmer edge thickness increased. For the case of $t_{e}=160 \mu \mathrm{m}$ under a larger air velocity $\left(u_{g}=66.7\right.$ and $\left.83.0 \mathrm{~m} / \mathrm{s}\right)$, no liquid accumulation was observed. At the lower flowrate condition $\left(Q_{l}=0.8\right.$ $\left.\mathrm{cm}^{3} /(\mathrm{s} \cdot \mathrm{cm})\right)$, there was also no liquid accumulation at the prefilmer edge under even smaller air velocity $\left(u_{g}=32.7\right.$ and $50.0 \mathrm{~m} / \mathrm{s}$ ). This leads to decrease of the breakup length and increase of the breakup frequency. Therefore, the breakup length increased and the breakup frequency decreased by increasing prefilmer edge thickness. In addition, the atomization process at $t_{e}=160 \mu \mathrm{m}$ may be separated from those at other thicker edge thicknesses.

On the other hand, it was observed that increasing air velocity reduces the breakup length, and also increases the breakup frequency. An increase of air velocity causes stronger shear force due to a larger air velocity. This force results in the elongated liquid film and makes unstable it faster. Thus, it leads to earlier disintegration which causes increasing the breakup frequency.

The FFT analysis was conducted to quantitatively evaluate the effect of the prefilmer edge thickness on the breakup frequency. Figure 5 shows the power spectrum at each prefilmer edge thickness, when the air velocity is 32.7 or $66.7 \mathrm{~m} / \mathrm{s}$ at $Q_{l}=2.6 \mathrm{~cm}^{3} /(\mathrm{s} \cdot \mathrm{cm})$. For the constant air velocity, the increase of prefilmer edge thickness caused the transition of the maximal power spectrum to a lower frequency. This was also due to the increase of liquid accumulation volume which causes the elongation of the liquid film. Comparing the power spectrums at different air velocities for the same edge thickness, a distinct tendency was found that the breakup frequency increase with increasing air velocity. The peak spectrums for $u_{g}=32.7 \mathrm{~m} / \mathrm{s}$ were less than $100 \mathrm{~Hz}$, while the peak power spectrums for $u_{g}=66.7 \mathrm{~m} / \mathrm{s}$ were ranged between $100-300 \mathrm{~Hz}$.

Figure 6 shows the mean breakup length of the liquid film as function of the prefilmer edge thickness when $Q_{l}=0.8$ and $2.6 \mathrm{~cm}^{3} /(\mathrm{s} \cdot \mathrm{cm})$. As the liquid flowrate increases, the breakup length increases. For both liquid flow rates of 0.8 and $2.6 \mathrm{~cm}^{3} /(\mathrm{s} \cdot \mathrm{cm})$, the breakup length shows a linear increase with the prefilmer edge thickness. This tendency was found for all air velocity conditions. The larger errors for $u_{g}=16.6 \mathrm{~m} / \mathrm{s}$ were due to the elongated liquid film that exceed the field of view. Also, the film dry-out was observed upstream the prefilmer edge, and leads to the larger errors. The increase of liquid flow rate leads to an increase of the breakup length. Especially at larger prefilmer edge thicknesses such as 2000 and $3000 \mu \mathrm{m}$, the effect of the air velocity on the breakup length becomes larger. Due to the increase of the number of atomization events per second and the increase of the accumulated liquid volume, the area of the liquid film attaching to the prefilmer edge in the calculation domain increases at $Q_{l}=2.6$ $\mathrm{cm}^{3} /(\mathrm{s} \cdot \mathrm{cm})$. Thus, the mean breakup length becomes larger than that at $Q_{l}=0.8 \mathrm{~cm}^{3} /(\mathrm{s} \cdot \mathrm{cm})$. 

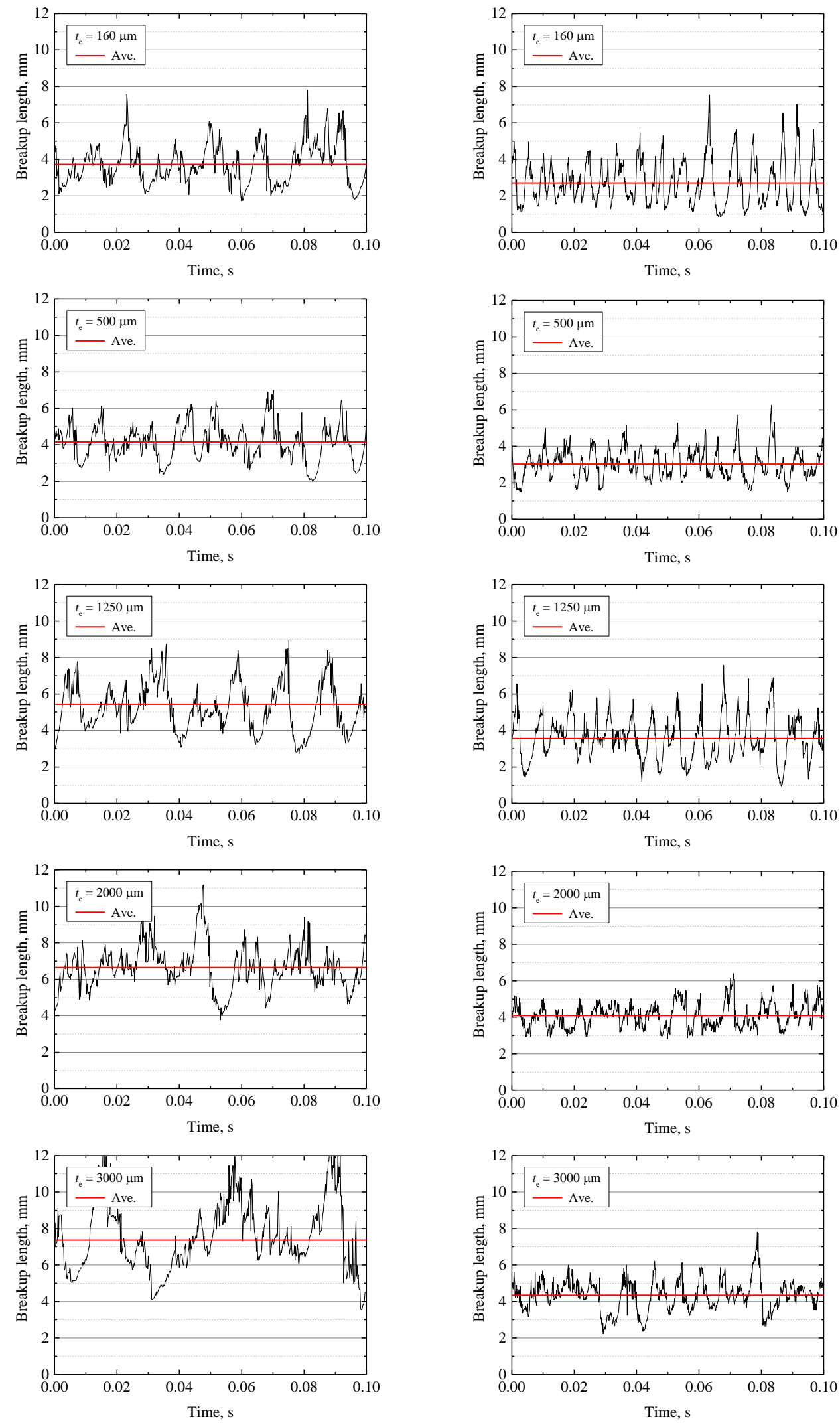

(a) $u_{g}=32.7 \mathrm{~m} / \mathrm{s}$

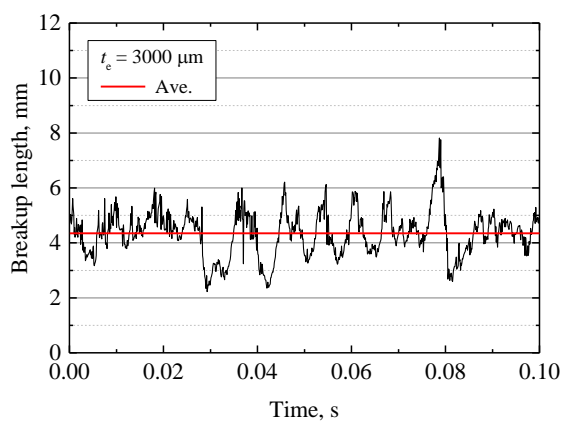

(b) $u_{g}=66.7 \mathrm{~m} / \mathrm{s}$

Figure 4. Time evolution of breakup length of liquid film at each prefilmer edge thickness. 

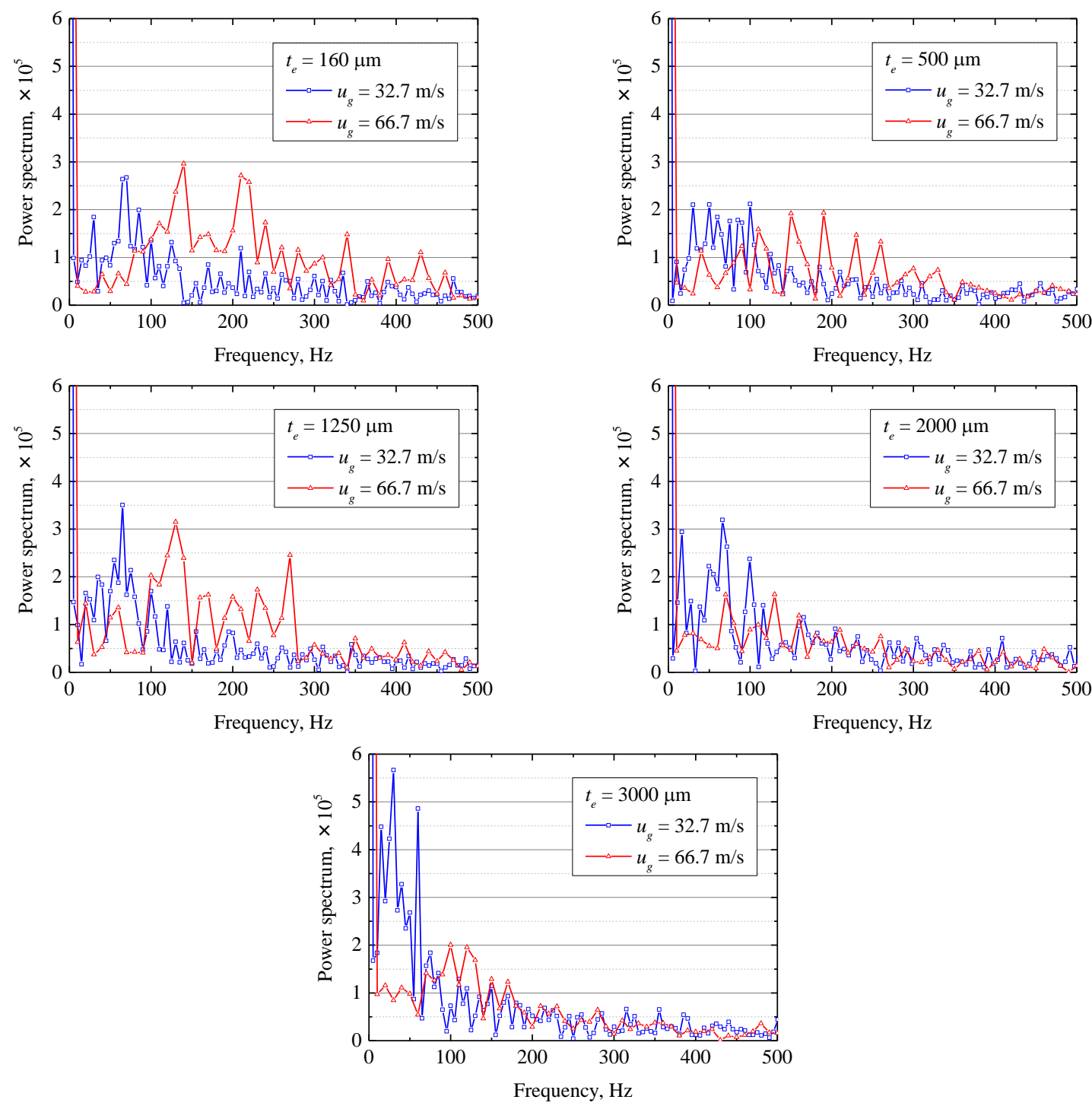

Figure 5. Variations of power spectrum with air velocity at each edge thickness of prefilmer at $Q_{l}=2.6 \mathrm{~cm}^{3} /(\mathrm{s} \cdot \mathrm{cm})$.
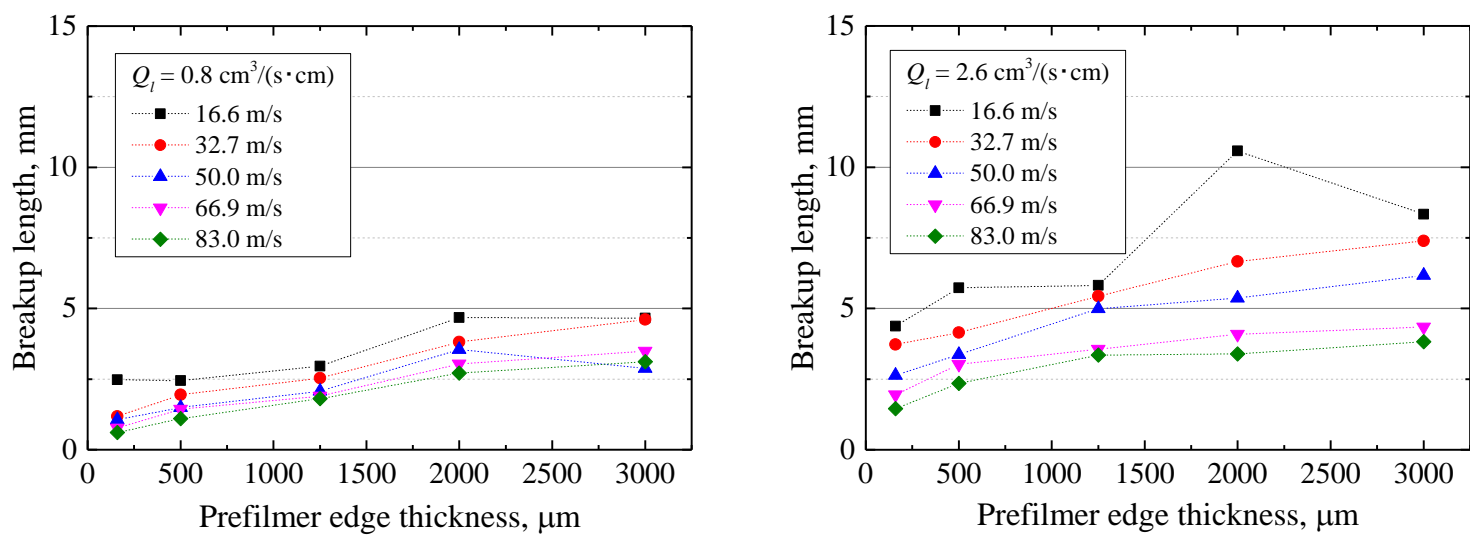

Figure 6. Mean breakup length of liquid film as function of prefilmer edge thickness. 
Fernandez et al. investigated the primary atomization of a liquid film generated by a planar airblast atomizer [7]. They obtained a correlation that the breakup length divided by the liquid film thickness is a function of the momentum flux ratio times the Weber number. To characterize the breakup length at various prefilmer edge thicknesses, we contain the thickness of the accumulated liquid at the prefilmer edge in the correlation as follows:

$$
\begin{aligned}
& L_{b} / t_{l}=\left[\left(u_{l} / u_{g}\right) /\left(M \cdot W e_{t l}\right)\right]^{1 / 3}, \\
& t_{l}=t_{e}+t_{p l}, \\
& W e_{t l}=\left(\rho t_{l} u_{l}\right) / \sigma,
\end{aligned}
$$

where $t_{l}$ is the thickness of the accumulated liquid at the edge, $t_{p l}$ is the liquid film thickness on the prefilmer. The liquid film thickness flowing over the prefilmer, $t_{p l}$, was measured by the contact needle method in our previous study [2]. The measured liquid film thicknesses are ranged from 55 to $600 \mu \mathrm{m}$ at each tested condition, depending on the air velocity. Inamura et al. revealed that the film thickness flowing on the prefilmer decreases as the air velocity increases [2]. At small air velocity, the film thickness increases with the liquid flowrate. At large air velocity, however, the effect of the liquid flowrate on the film thickness becomes smaller.

Figure 7 shows the dimensionless breakup length of the liquid film. The results plotted in Figure 7 show that there was a correlation between the dimensionless breakup length and $\left[\left(u_{l} / u_{g}\right) /\left(M \cdot W e_{t}\right)\right]^{1 / 3}$. However, the data surrounded by a red line strays off the solid line. The solid line in the figure shows the line which was approximated by a linear approximation with the data measured at all experimental conditions except those at a thinner prefilmer edge thickness $\left(t_{e}=160\right.$, and $\left.500 \mu \mathrm{m}\right)$ at $Q_{l}=2.6 \mathrm{~cm}^{3} /(\mathrm{s} \cdot \mathrm{cm})$ surrounded by a red line. This could be due to the ratio of prefilmer thickness to the liquid film thickness flowing over the prefilmer. For the cases when $t_{e}=160$, and $500 \mu \mathrm{m}$, the edge thickness is of the same order of the liquid film thickness flowing over the prefilmer. A sensitivity to the liquid film thickness is relatively large compared to the case of a thicker edge thickness. Thus, the dimensionless breakup length can be easily affected by the experimental error in the liquid film thickness. In fact, the liquid film was measured at $2 \mathrm{~mm}$ upstream from the prefilmer edge, because it is difficult to exactly measure the liquid film thickness of the accumulated liquid film at the edge by the contact needle method used in this study. It may also be that the atomization process is separated from other cases of the prefilmer edge thickness. In fact, no liquid accumulation was only observed at a thinner prefilmer edge thickness. Further research efforts are needed to clarify this issue.

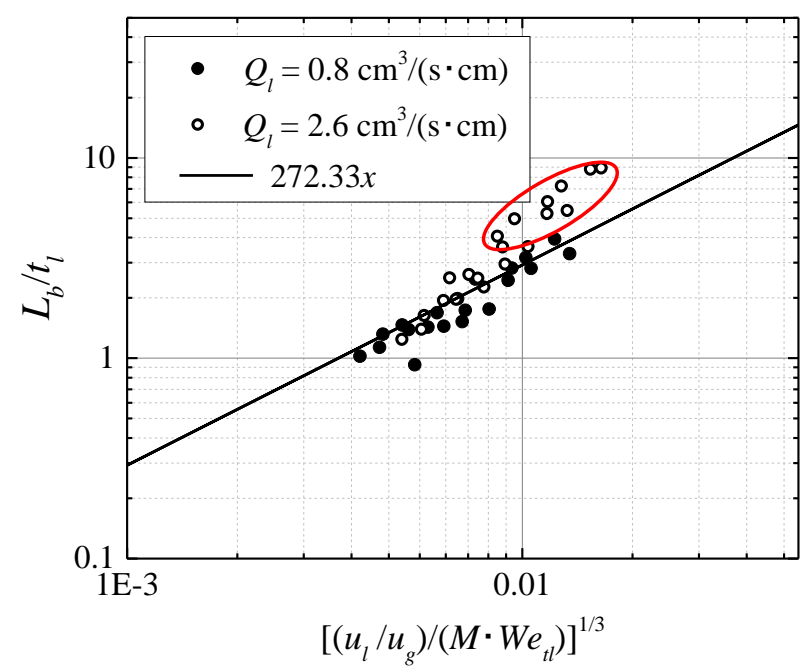

Figure 7. Dimensionless breakup length of liquid film.

\section{Conclusions}

In this study, the effect of widely varying prefilmer edge thickness $(160,500,1250,2000$, and $3000 \mu \mathrm{m})$ on the breakup phenomena of the liquid film in the prefilming airblast atomizer was experimentally investigated. The conclusions obtained in this study are as follows:

1. To quantitatively evaluate complicated breakup process of a liquid film at the prefilmer edge, the image processing method to determine the spatially-averaged breakup length and frequency based on the area of a liquid film and the prefilmer width has been developed. 
2. In a prefilming airblast atomizer, the liquid accumulation attaching to the prefilmer edge has a significant impact on the breakup process of a liquid film. As the prefilmer edge thickness increases, the liquid accumulation volume increases. A larger liquid accumulation causes the increase of the breakup length of the liquid film and the decrease of the breakup frequency.

3. For the case of $t_{e}=160 \mu \mathrm{m}$ at larger air velocity $\left(u_{g}=66.7\right.$ and $\left.83.0 \mathrm{~m} / \mathrm{s}\right)$, no liquid accumulation was observed. Also, at the lower flowrate condition $\left(Q_{l}=0.8 \mathrm{~cm}^{3} /(\mathrm{s} \cdot \mathrm{cm})\right)$, there was no liquid accumulation at the prefilmer edge at even smaller air velocity $\left(u_{g}=32.7\right.$ and $\left.50.0 \mathrm{~m} / \mathrm{s}\right)$. It could be that the breakup process at a thinner prefilmer edge thickness can be separated from that at a thicker one.

4. The FFT analysis indicated that the increase of prefilmer edge thickness causes the transition of the maximal power spectrum to a lower frequency, because of the increase of liquid accumulation volume which causes the elongation of the liquid film. The peak spectrums for $u_{g}=32.7 \mathrm{~m} / \mathrm{s}$ were less than $100 \mathrm{~Hz}$, while the peak power spectrums for $u_{g}=66.7 \mathrm{~m} / \mathrm{s}$ were ranged between $100-300 \mathrm{~Hz}$. These tendency were found in all prefilmer thicknesses.

5. The breakup length of a liquid film is expressed by the following empirical equation which contains the effect of the prefilmer edge thickness:

$$
\frac{L_{b}}{t_{l}}=272.33\left(\frac{u_{l} / u_{g}}{M \cdot W e_{t l}}\right)^{\frac{1}{3}}
$$

\section{Nomenclature}

$A_{b} \quad$ Area of liquid film $\left[\mathrm{mm}^{2}\right]$

$f_{b} \quad$ Breakup frequency $[\mathrm{Hz}]$

$L_{b} \quad$ Breakup length $[\mathrm{kg}]$

$L_{e} \quad$ Width of prefilmer edge [mm]

$M \quad$ Momentum flux ratio [-]

QI Liquid flow rate $\left[\mathrm{cm}^{3} /(\mathrm{s} \cdot \mathrm{cm})\right]$

$t_{e} \quad$ Prefilmer edge thickness [ $\left.\mu \mathrm{m}\right]$

$t_{\text {I }} \quad$ Accumulated liquid film thickness $[\mu \mathrm{m}]$ $t_{p l} \quad$ Liquid film thickness on prefilmer $[\mu \mathrm{m}]$

$u_{g} \quad$ Gas velocity [m/s]

$u_{1} \quad$ Liquid velocity $[\mathrm{m} / \mathrm{s}]$

$w \quad$ Width of prefilmer edge [mm]

We Webber number [-]

$\rho \quad$ Density $\left[\mathrm{kg} / \mathrm{m}^{3}\right]$

$\sigma \quad$ Surface tension $[\mathrm{N} / \mathrm{m}]$

\section{References}

[1] Lefebvre, A. H., 1980, Progress in Energy and Combustion Science, 6 (3), pp. 233-261.

[2] Inamura, T., Shirota, M., Tsushima, M., Kato, M., Hamajima, S., and Sato, A., Sep. 2-6, 2012, $12^{\text {th }}$ Triennial International Conference on Liquid Atomization and Spray Systems.

[3] Gepperth, S., Müller, A., Koch, R., and Bauer, H. -J., Sep. 2-6, 2012, $12^{\text {th }}$ Triennial International Conference on Liquid Atomization and Spray Systems.

[4] Tsushima, M., Yamashita, K., Sato, A., Inamura, T., and Shirota, M., Dec. 17-18, 2009, 18th ILASS-Japan Symposium (in Japanese).

[5] Koch, R., Braun, S., Wieth, L., Chaussonnet, G., Dauch, T., and Bauer, H. -J., 2017, European Journal of Mechanics B/Fluids, 61, pp. 271-278.

[6] Sattelmayer, T., and Wittig, S., 1986, Jornal of Engineering for Gas Turbines and Power, 108, pp. 465-472.

[7] Fernandez, V. G., Berthoumie, P., Lavergne, G., 2009, C. R. Mecanique, 337, pp. 481-491. 